\title{
Histopathological assessment of bleeding from polyps of the colon and rectum
}

\author{
A WALDOCK,* I O ELLIS, * N C M ARMITAGE, $\dagger$ D R TURNER, ${ }^{*}$ J D HARDCASTLE $\dagger$ \\ From the Departments of *Histopathology and $\uparrow$ Surgery, Queen's Medical Centre, Nottingham
}

SUMMARY One hundred and twenty seven colorectal polyps were examined to assess histopathological evidence of recent and old haemorrhage to test the usefulness of faecal occult blood tests in detecting colorectal neoplasia, in particular premalignant adenomas. Evidence of haemorrhage was consistently found in adenomas but was rare in non-neoplastic polyps. Haemorrhage within adenomas was predominantly stromal and associated with dilated, congested vessels. Factors associated with more severe haemorrhage were size, pedunculation, and villous growth; the degree of epithelial dysplasia and the age and sex of the patient were not associated factors. Proximal polyps showed more old haemorrhage than rectal polyps, but there was no such difference for recent haemorrhage.

Faecal occult blood tests have been investigated as a screening method for colorectal cancer. The test, devised by Greegor ${ }^{1}$ entails smearing a faecal sample on a slide impregnated with guaiac. This contains a phenolic compound which undergoes a colour change when developed with hydrogen peroxide if haemoglobin is present within the smear. It is a fairly cheap and simple method of detecting carcinomas as well as a proportion of adenomas. Published findings on this method have recently been reviewed by Simon. ${ }^{2}$ Although there has been some work on the pattern of bleeding in adenomas, as shown by radiochromiumlabelled erythrocytes, ${ }^{34}$ we are only aware of one study that has investigated histopathological changes associated with bleeding in colorectal neoplasia. ${ }^{5}$

\section{Material and methods}

One hundred and twenty seven colorectal polyps were chosen at random from the files of the Nottingham Colorectal Cancer Screening Study. Of these, 80 were adenomas from "asymptomatic" patients (adenomas detected due to positive faecal occult blood tests), 24 were adenomas from patients presenting with symptoms, and 23 were non-neoplastic polyps (12 metaplastic, 11 inflammatory). All polyps were fixed, measured, embedded in paraffin wax, processed routinely and sectioned at $5 \mu \mathrm{m}$. One set of sections was stained with haematoxylin and eosin to evaluate the degree of

Accepted for publication 12 December 1988 recent haemorrhage, while another set was stained with Perls's Prussian blue to detect the presence of haemosiderin pigment indicating past haemorrhage.

The sex and age of patients from whom the polyps were removed, were as follows: 71 men, aged 49-84 years (mean 64); 56 women, aged 54-82 years (mean 69). The polyps were typed histologically according to the criteria of the World Health Organization ${ }^{6}$ and were described morphologically according to the criteria of Morson. ${ }^{7}$ The grading of the extent of epithelial dysplasia was based on the criteria of Konishi and Morson ${ }^{8}$ and Brown et al, ${ }^{9}$ and included both the most prominent and the severest atypia present within the adenoma.

The degree of recent haemorrhage was recorded on a scale of $4+$ if grossly visible on the slide, $3+$ if extensively visible with the $\times 4$ objective, $2+$ if moderately visible with the $\times 4$ objective, $1+$ if moderately visible with the $\times 10$ objective and trace if only just visible with the $\times 10$ objective. For tabulation purposes these categories were reduced to three: mild haemorrhage comprising trace and $1+$; moderate haemorrhage comprising $2+$; and severe haemorrhage comprising $3+$ and $4+$. The degree of haemosiderin content was recorded in a similar manner and these categories were also reduced to three: no haemosiderin comprising 0 ; moderate haemosiderin comprising $1+$ and $2+$; pronounced haemosiderin comprising $3+$ and $4+$. The data were analysed by the $\chi^{2}$ test to assess the association tables for significance $(p<0.05)$ and some were analysed further using the Kendall rank correlation coefficient. 


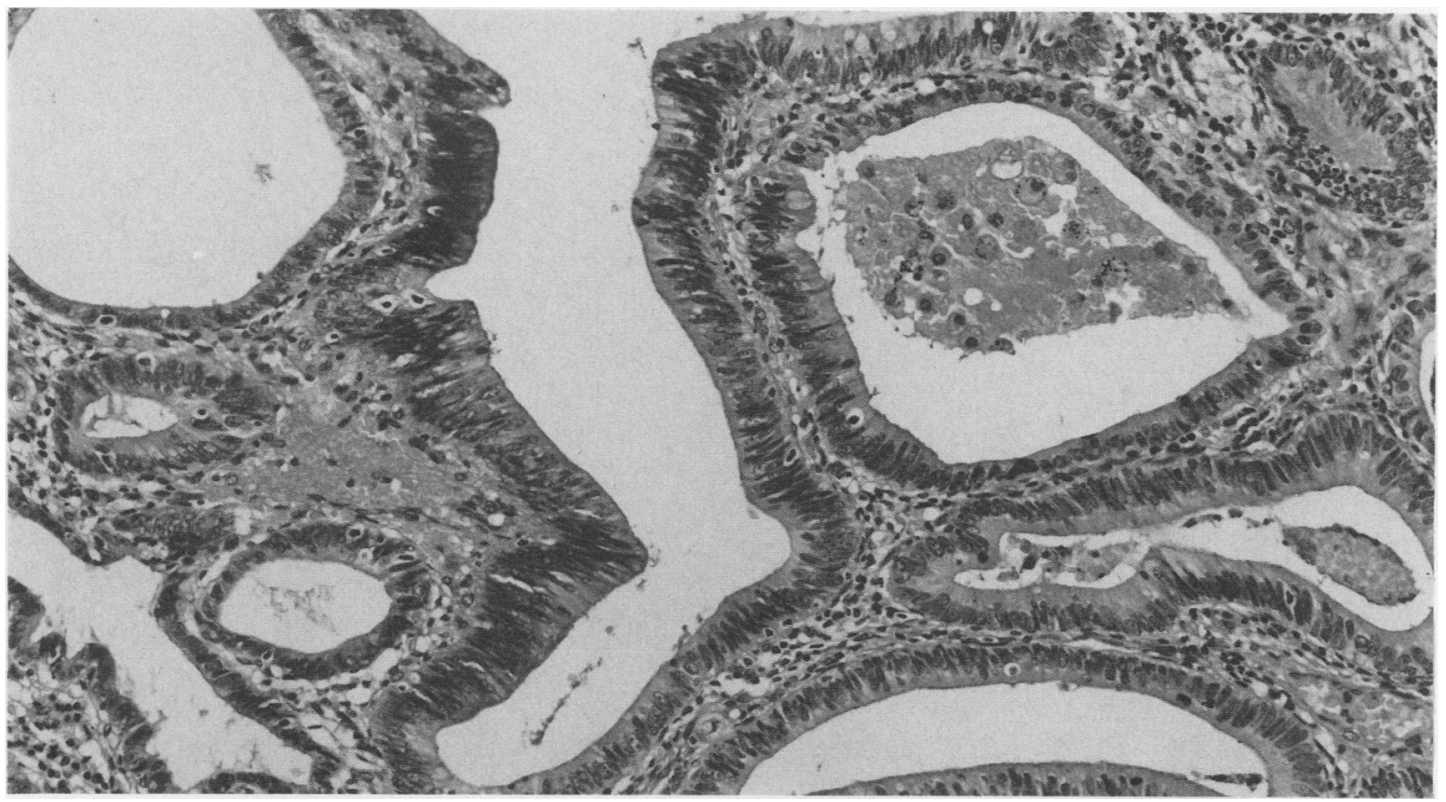

Fig 1 Tubular adenoma showing pronounced extravasation of red blood cells into lamina propria and lumen of gland.

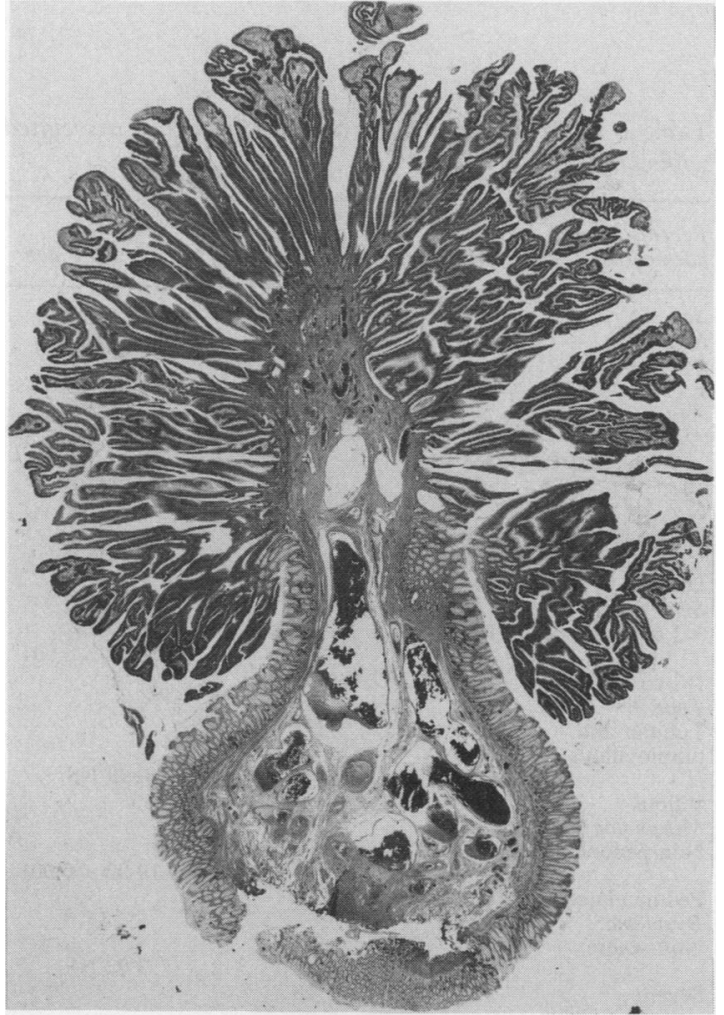

Fig 2 Moderately large tubulovillous adenoma showing prominent dilated vessels in submucosa of the pedicle.

\section{Results}

Stromal haemorrhage was the predominant pattern of recent haemorrhage seen in all types of adenoma studied. In larger tubular adenomas recent haemorrhage was seen in gland lumina as well as in the lamina propria (fig 1). Haemorrhage was associated with dilated and congested vessels, especially in the stalks of pedunculated adenomas (fig 2). Tubulovillous and villous adenomas often showed evidence of trauma with damage to villi and associated haemorrhage. Surface erosion and ulceration were rarely seen. Evidence of old haemorrhage was seen in similar sites to those seen for recent haemorrhage but was generally less prominent (fig 3).

Sixty eight $(85 \%)$ of asymptomatic adenomas and $18(75 \%)$ of symptomatic adenomas showed a moderate or severe degree of recent haemorrhage, compared with only $6(26 \%)$ of non-neoplastic polyps $(p<0.01)$ (table 1). Fifty seven $(71 \%)$ of asymptomatic adenomas showed some haemosiderin compared with $11(46 \%)$ of symptomatic adenomas $(p<0.05)$ and $3(13 \%)$ of non-neoplastic polyps $(\mathrm{p}<0.05)($ table 1$)$.

The various factors associated with colorectal adenomas were compared with the degree of recent haemorrhage (table 2) and the degree of haemosiderin content (table 3). Neither the age and sex of the patient, nor the site and type of the lesion were indicators of recent haemorrhage. Thirty five $(87 \%)$ of adenomas measuring greater than $1 \mathrm{~cm}$, however, 


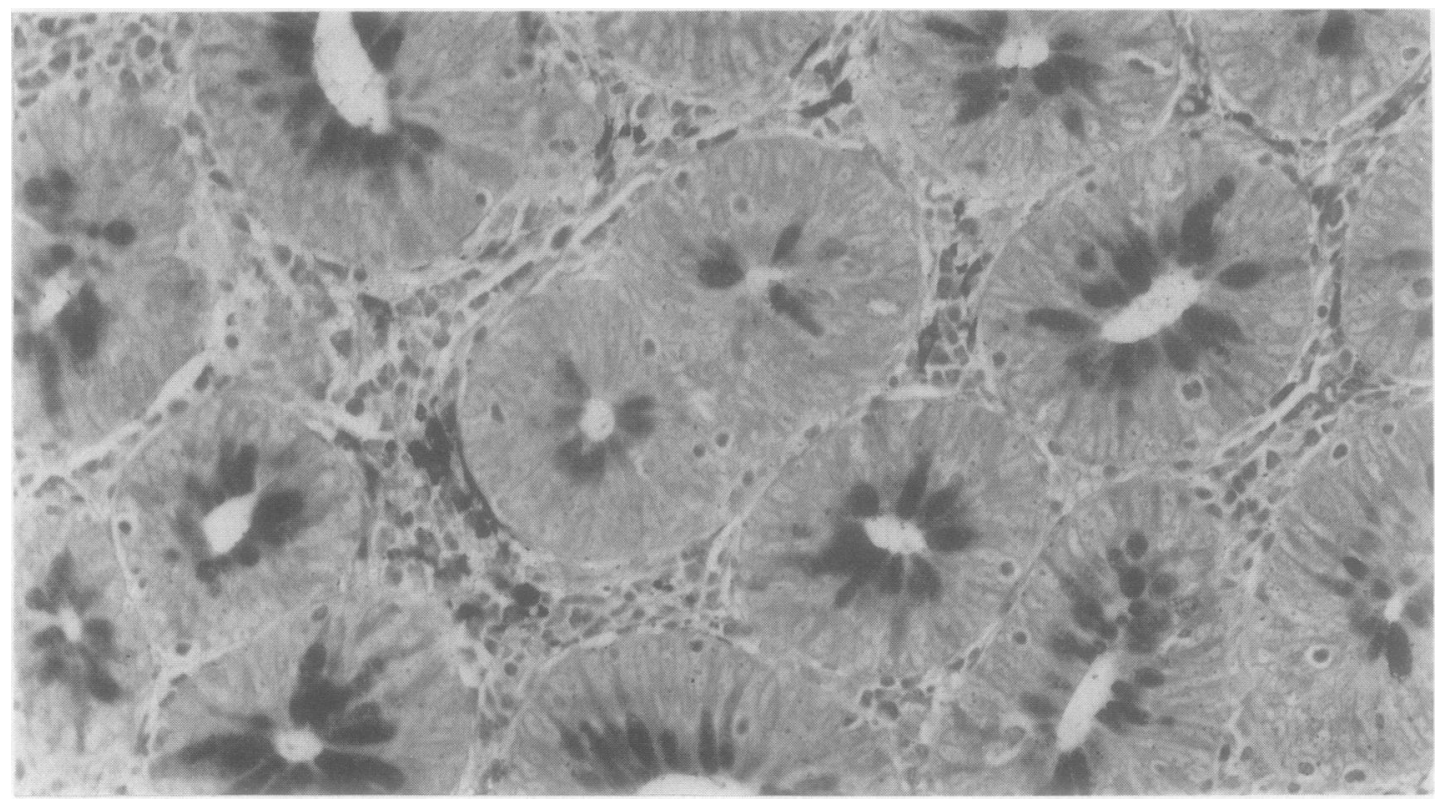

Fig 3 Distribution of haemosiderin pigment indicative of old haemorrhage is similar to that seen for recent haemorrhage. In this adenoma there is a considerable amount of haemosiderin pigment present in the lamina propria (Perls's stain).

showed severe recent haemorrhage compared with 26 $(41 \%)$ of those measuring $1 \mathrm{~cm}$ or less (Kendall rank correlation coefficient, $\mathrm{p}<0.001)$. Morphological (macroscopic type) analysis showed that $24(96 \%)$ of pedunculated adenomas showed severe recent haemorrhage compared with $37(47 \%)$ for other types of lesions $(p<0.001)$. The degree of epithelial dysplasia within adenomas failed to be an indicator of recent haemorrhage, even though $18(72 \%)$ of severely dysplastic adenomas showed a pronounced degree of recent haemorrhage.

Table 3 shows that neither the sex and age of the patient nor the degree of epithelial dysplasia were indicators of haemosiderin content. Five $(50 \%)$ of lesions, in the ascending or transverse colon, however,

Table 1 Haemorrhage in colorectal lesions

\begin{tabular}{lrrr}
\hline & Mild & Moderate & Severe \\
\hline Recent haemorrhage: & & & \\
Asymptomatic adenomas (\%) & $12(15)$ & $18(23)$ & $50(62)$ \\
Symptomatic adenomas (\%) & $6(25)$ & $7(29)$ & $11(46)$ \\
Non-neoplastic polyps (\%) & $17(74)$ & $3(13)$ & $3(13)$ \\
& & & \\
Past haemorrhage & & & \\
(haemosiderin) & & & \\
Asymptomatic adenomas (\%) & $23(29)$ & $37(46)$ & $20(25)$ \\
Symptomatic adenomas (\%) & $13(54)$ & $7(30)$ & $4(16)$ \\
Non-neoplastic polyps (\%) & $20(87)$ & $3(13)$ & $0(0)$ \\
\hline
\end{tabular}

Table 2 Recent haemorrhage and various factors associated with colorectal adenomas

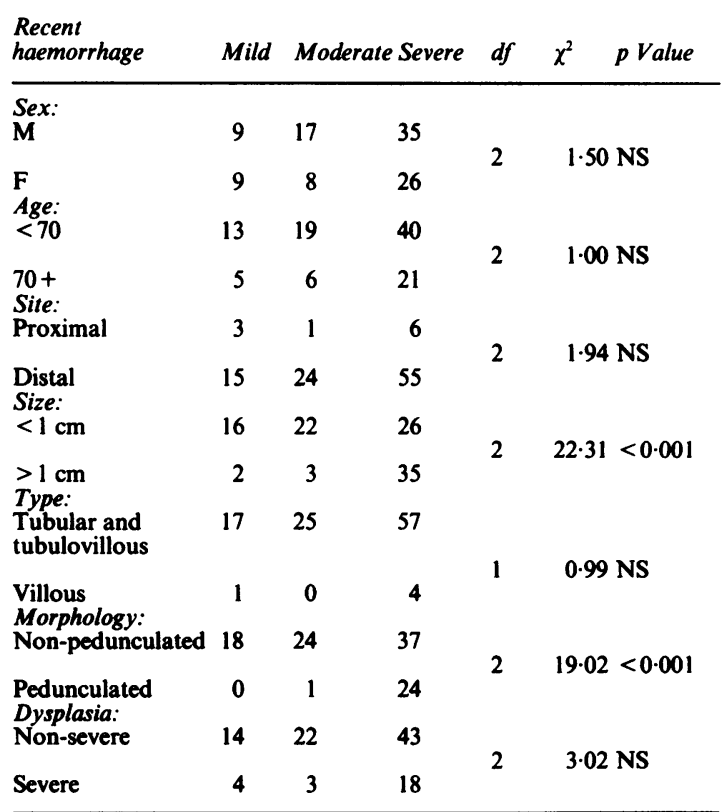

Proximal $=$ ascending colon and transverse colon.

Distal = descending and sigmoid colon and rectum. 
Table 3 Old haemorrhage and various factors associated with colorectal adenomas

\begin{tabular}{|c|c|c|c|c|c|c|}
\hline $\begin{array}{l}\text { Old } \\
\text { haemorrhage }\end{array}$ & Mild & Moderate & e Severe & $d f$ & $x^{2}$ & p Value \\
\hline $\begin{array}{l}\text { Sex: } \\
\text { M }\end{array}$ & 22 & 23 & 16 & 2 & \multicolumn{2}{|c|}{$1.46 \mathrm{NS}$} \\
\hline $\begin{array}{l}\mathrm{F} \\
\text { Age: }\end{array}$ & 14 & 21 & 8 & & & \\
\hline $\begin{array}{l}\text { Age. } \\
<70\end{array}$ & 25 & 28 & 19 & \multirow[t]{2}{*}{2} & \multicolumn{2}{|c|}{$1.76 \mathrm{NS}$} \\
\hline $\begin{array}{l}70+ \\
\text { Site: }\end{array}$ & 11 & 16 & 5 & & & \\
\hline $\begin{array}{l}\text { Proximal colon } \\
\text { Distal colon } \\
\text { Rectum }\end{array}$ & $\begin{array}{r}2 \\
17 \\
17\end{array}$ & $\begin{array}{r}3 \\
20 \\
21\end{array}$ & $\begin{array}{r}5 \\
16 \\
3\end{array}$ & 2 & $11 \cdot 3$ & $33<0.01$ \\
\hline $\begin{array}{l}\text { Size: } \\
<1 \mathrm{~cm}\end{array}$ & 28 & 29 & 7 & 2 & \multicolumn{2}{|c|}{$14.99<0.001$} \\
\hline $\begin{array}{l}>1 \mathrm{~cm} \\
\text { Type: } \\
\text { Tubular and } \\
\text { tubulovillous }\end{array}$ & $\begin{array}{r}8 \\
32\end{array}$ & $\begin{array}{l}15 \\
43\end{array}$ & $\begin{array}{l}17 \\
24\end{array}$ & \multirow[b]{2}{*}{1} & \multirow{2}{*}{\multicolumn{2}{|c|}{$4.78<0.05$}} \\
\hline $\begin{array}{l}\text { Villous } \\
\text { Morphology: }\end{array}$ & 4 & 1 & 0 & & & \\
\hline Non-pedunculated & 33 & 35 & 11 & \multirow{2}{*}{2} & \multirow{2}{*}{\multicolumn{2}{|c|}{$17 \cdot 10<0.001$}} \\
\hline $\begin{array}{l}\text { Pedunculated } \\
\text { Dysplasia: }\end{array}$ & 3 & 9 & 13 & & & \\
\hline Non-severe & 27 & 35 & 17 & \multirow[t]{2}{*}{2} & \multirow{2}{*}{\multicolumn{2}{|c|}{$0.67 \mathrm{NS}$}} \\
\hline Severe & 9 & 9 & 7 & & & \\
\hline
\end{tabular}

Proximal $=$ ascending colon and transverse colon.

Distal $=$ descending and sigmoid colon.

showed a pronounced degree of haemosiderin content compared with only three $(7 \%)$ of lesions situated in the rectum $(p<0.01)$. Seventeen $(42 \%)$ of adenomas greater than $1 \mathrm{~cm}$ in diameter showed a pronounced degree of haemosiderin deposition compared with seven $(11 \%)$ of those measuring $1 \mathrm{~cm}$ or less (Kendall rank correlation coefficient $\mathrm{p}<0.001$ ). Sixty seven $(68 \%)$ of tubulovillous and tubular adenomas showed signs of haemosiderin content compared with only one $(20 \%)$ of villous adenomas $(p<0.05)$. Finally, 13 $(52 \%)$ of pedunculated adenomas showed a pronounced degree of haemosiderin deposition compared with $11(14 \%)$ for the other types of lesion $(p<0.001)$.

\section{Discussion}

The major pattern of haemorrhage in adenomas shown in this study was stromal rather than glandular, and was associated with dilated, congested vessels, which supports the findings of previous work. ${ }^{5}$ Nevertheless, evidence of a substantial degree of trauma, due to luminal contents was also observed. This was particularly true in association with the villous fronds of tubulovillous and villous adenomas, resulting in fracture of the fronds and severe haemorrhage. Even so, this mechanism of haemorrhage may be different to that seen in carcinomas, which are thought to bleed mainly by surface erosion in areas of tissue necrosis. ${ }^{5}$

Adenomas were associated with moderate or severe degrees of recent haemorrhage and haemosiderin content, compared with those types of non-neoplastic polyp studied (metaplastic and inflammatory). This is probably not due to the neoplastic nature of adenomas, but to the fact that most non-neoplastic polyps are less than $5 \mathrm{~mm}$ in size. This suggests that nonneoplastic polyps are less likely to be detected by the faecal occult blood test and thus increase its predictive value for neoplasia. It was also observed that the degree of epithelial dysplasia is no indicator of recent or old haemorrhage, thereby suggesting that the faecal occult blood test is no indicator of malignant potential within colorectal adenomas.

Half of the adenomas from the ascending and transverse colon showed pronounced haemosiderin content while adenomas from the rectum showed considerably less deposition. These findings differ from those of Sobin ${ }^{5}$ and may be a reflection of the differing methods of selection of the adenomas studied. Alternatively, it may be that adenomas in the proximal colon have bled several times before they are detected by faecal occult blood testing.

Villous adenomas tend to have more evidence of recent haemorrhage than tubular and tubulovillous adenomas, even though these data did not reach significance. Tubular and tubulovillous adenomas, however, are more likely to show some signs of old haemorrhage. Villous adenomas predominantly occur in the sigmoid colon and rectum, where surface abrasion from faecal matter is both continuous and intense. This results in more frequent luminal haemorrhage, little stromal haemorrhage, and consequently little or no haemosiderin deposition. Pedunculated adenomas are associated with a pronounced degree of recent haemorrhage and haemosiderin deposition, which is likely to be due to the twisting and bending to which these adenomas are subjected, causing vascular compression, venous congestion, and subsequent leakage of blood from stress points. The size of the adenoma is directly correlated to both the degree of recent haemorrhage and the extent of haemosiderin deposition, which agrees with previous pathological ${ }^{5}$ and clinical studies. ${ }^{4}$ In general, adenomas which are prone to haemorrhage are likely to incur frequent episodes of haemorrhage albeit intermittently. ${ }^{10}$

Although our observations indicate that all adenomas studied showed some degree of recent haemorrhage, some adenomas may not bleed during the period of testing and hence faecal occult blood will not be detected by using the faecal occult blood test. This should be considered when assessing the effectiveness of this test, which is not only limited by the technical 
aspects of obtaining good sensitivity and specificity, but more importantly by the extent of the haemorrhage from the adenomas.

We are grateful to $\mathrm{Mr} \mathrm{W}$ Brackenbury for the photography and Mrs $\mathbf{J}$ Rainbow for preparation of the manuscript.

\section{References}

1 Greegor DH. Diagnosis of large bowel cancer in the asymptomatic patient. JAMA 1967;201:943-5.

2 Simon JB. Occult blood screening for colorectal carcinomas: a critical review. Gastroenterology 1985;88:820-37.

3 Herzog P, Holtermuller KH, Preiss J, et al. Faecal blood loss in patients with colonic polyps: a comparison of measurements with 51 chromium-labelled erythrocytes and with the Haemoccult test. Gastroenterology 1982;83:957-62.

4 Macrae FA, St John DJB. Relationship between patterns of bleeding and haemoccult sensitivity in patients with colorectal
5 Sobin LH. The histopathology of bleeding from polyps and carcinomas of the large intestine. Cancer 1985;55:577-81.

6 Morson BC, Sobin LH. Histological typing of intestinal tumours. International histological classification of tumours, No 15. Geneva: World Health Organization, 1976.

7 Morson BC. The pathogenesis of colorectal cancer. Major problems in pathology. Vol 10. Philadelphia: WB Saunders Company, 1978.

8 Konishi F, Morson BC. Pathology of colorectal adenomas: a colonoscopic survey. J Clin Pathol 1982;35:830-41.

9 Brown LJR, Smeeton NC, Dixon MF. Assessment of dysplasia in colorectal adenomas: an observer variation and morphometric study. J Clin Pathol 1985;38:174-9.

10 Greegor DH. Occult blood testing for the detection of asymptomatic colon cancer. Cancer 1971;28:131-4.

Requests for reprints to: Dr I O Ellis, Department of Histopathology, Queen's Medical Centre, Nottingham NG7 2UH, England. 